\title{
ARTICLE
}

\section{A study of cerium extraction kinetics by TODGA in acidified and non-acidified organic solvent phases in the context of fission product management}

\author{
Michael A. Bromley and Colin Boxall* \\ Lancaster University, Engineering, Gillow Avenue, Lancaster, LA1 4YW, U.K.
}

\begin{abstract}
In the context of management of the waste streams arising from the reprocessing of spent nuclear fuel, studies have been conducted into the solvent extraction kinetics of lanthanide fission products by the oxygen-donor ligand N,N,N',N'-tetraoctyl diglycolamide (TODGA) and the effects of solvent phase acidity on the effectiveness of this organic extractant molecule. In this study, we observe a reduction in the rate of cerium extraction as solvent phase acidity increases, attributed to aggregation of the extractant. The effect is shown to be reversible through neutralization while a permanent prevention method is the subject of further work.
\end{abstract}

Keywords: TODGA; rotating diffusion cell; nuclear; separation; extraction; reprocessing

\section{Introduction}

Nuclear power is of great importance to the future of low carbon energy production and the ability to separate and recover the actinide elements from spent fuel is a key requirement for a sustainable nuclear fuel cycle. While the extraction of $\mathrm{U}$ and $\mathrm{Pu}$ for the fabrication of new fuel is well established with the PUREX process [1], recovery of the minor actinides, and particularly their separation from the chemically similar lanthanides, remains challenging.

A range of organic extractant molecules, such as N,N,N',N'-tetraoctyl diglycolamide (TODGA), have been developed for the recovery of trivalent actinides through solvent extraction processes with particular aims to achieve effective recycling of minor actinides from long-lived radioactive waste. Consequently, it is important to develop a comprehensive understanding of the extractant molecules and diluents as well as the associated chemical extraction mechanisms and kinetics involved in such processes. A more complete understanding will enable future process optimisation and assessment of the long-term suitability of proposed extractants and their potential for regeneration / recycling in order to minimize process waste and cost.

As such, studies of the interfacial and mass transport kinetics of the extraction of trivalent lanthanide fission products (as minor actinide surrogates) from aqueous $\mathrm{HNO}_{3}$ solutions by the organic extractant molecule TODGA in organic solvent systems, such as dodecane, have been ongoing in our laboratories with the use of a rotating diffusion cell (RDC) [2].

The RDC is a compartmental diffusion apparatus

*Corresponding author. Email: c.boxall@lancaster.ac.uk consisting of two solution vessels separated by a Millipore GSWP04700 nitrocellulose membrane filter, Figure 1. This creates a defined solution phase interface across which the transport of species may be studied.

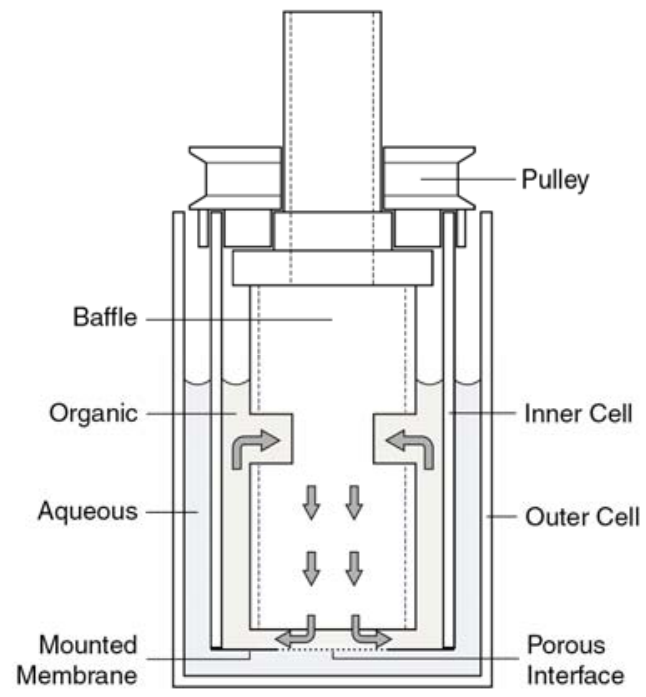

Figure 1. Schematic diagram of the rotating diffusion cell.

Rotation of the inner RDC vessel generates hydrodynamic flow conditions at each side of the interface membrane [2]; at the outer membrane surface, these conditions closely match those of widely studied rotating disc systems while in the inner vessel, a flow pattern towards and normal to the interface is maintained via a static baffle, Figure 1.

Establishment of a robust and controllable hydrodynamic environment at each side of the solution phase interface enables the study of interfacial kinetics 
and the interrogation of several kinetic regimes from mass transport to thermodynamic control as well as the transition between the two [3].

Through use of this technique, we have quantified the interfacial kinetics of the extraction of $\mathrm{Ce}(\mathrm{III})$ by TODGA [4] and have identified that the key Ce-TODGA complexation reaction takes place in the aqueous solution phase close to the aqueous-organic interface [5]. Further observations include the linear dependence of the rate of $\mathrm{Ce}$ extraction on the aqueous concentrations of both $\mathrm{Ce}(\mathrm{III})$ and $\mathrm{HNO}_{3}$ (not shown), indicating the inclusion of a single nitrate ion in the first coordination sphere of the Ce(III)-TODGA complex; and, through variation of both hydrodynamic conditions and key solution concentrations, that the system operates under mixed mass transfer with chemical reaction (MTWCR) control [6]. These observations are in agreement with concurrent studies by Lelias et al and Simonin et al [4].

We also observe a reduction in the extraction kinetics of the $\mathrm{Ce}$ (III) / TODGA system where the organic solution phase is acidified both as a consequence of pre-contacting of the organic solution phase with an acidic aqueous phase, and from the increase in organic phase acidity due to simultaneous extraction of $\mathrm{HNO}_{3}$ with Ce(III) by TODGA within the RDC.

Given the acidic environments associated with solvent extraction processes, further investigation into the effects of solvent phase acidity on the extraction properties of TODGA form the basis for this paper.

\section{Experimental}

All reagents used are AnalaR grade or higher, and purchased from Sigma-Aldrich (Dorset, UK) or Alfa Aesar (Lancashire, UK) with the exception of TODGA (Technocomm Ltd., Falkland, UK). All water used is ultrapure doubly de-ionized water from a Direct-Q 3 UV Millipore water purification system (Millipore, Watford, UK) to a resistivity of $18.2 \mathrm{M} \Omega \cdot \mathrm{cm}$.

RDC studies are performed with a freshly prepared aqueous solution phase of $10 \mathrm{mmol} / \mathrm{dm}^{3} \mathrm{Ce}$ (III) $\mathrm{N}_{3} \mathrm{O}_{9}$ in $1 \mathrm{~mol} / \mathrm{dm}^{3} \mathrm{HNO}_{3}$ in the outer vessel and an organic solution phase of $0.2 \mathrm{~mol} / \mathrm{dm}^{3}$ TODGA in n-dodecane with $5 \%$ vol 1 -octanol in the inner vessel. Where organic solution phases are to be used in a non-equilibrated state, no prior contact is made with the aqueous phase. Where the organic phase is to be pre-equilibrated, contacting is performed via $15 \mathrm{~min}$ shaking in an Eppendorf ThermoMixer C (Eppendorf UK Ltd, Stevenage, UK) and 5 min centrifugation in a MicroStar 12 centrifuge (VWR, West Sussex, UK).

In the RDC, the nitrocellulose membrane is fully permeated with the organic solution phase, ensuring that the phase boundary is positioned at the outer membrane surface while equal solution levels in each vessel avoid any hydrostatic pressure effects across the interface. The $\mathrm{RDC}$ is then operated at constant rotation speed of $2 \mathrm{~Hz}$ for up to $240 \mathrm{~min}$ with monitoring of each solution phase performed using a Gamry Spectro-115U UV-Vis spectrophotometer (Gamry Instruments, Cheshire, UK).

Validation of spectrophotometric measurement of $[\mathrm{Ce}(\mathrm{III})]$ in the organic solvent phase was performed by contacting fresh organic phase solutions with a range of known [Ce(III)] aqueous solutions. The organic phase absorbance at $346 \mathrm{~nm}$ was plotted against the quantified depletion of $\mathrm{Ce}$ (III) in the aqueous phase, as determined via both spectrophotometry and via ion chromatography with conductivity detection, Figure 2. These plots enable molar extinction coefficients for $[\mathrm{Ce}(\mathrm{III})]_{\mathrm{org}}$ of $0.0090 \mathrm{~m}^{2} / \mathrm{mol}$ and $0.0094 \mathrm{~m}^{2} / \mathrm{mol}$ to be determined respectively.

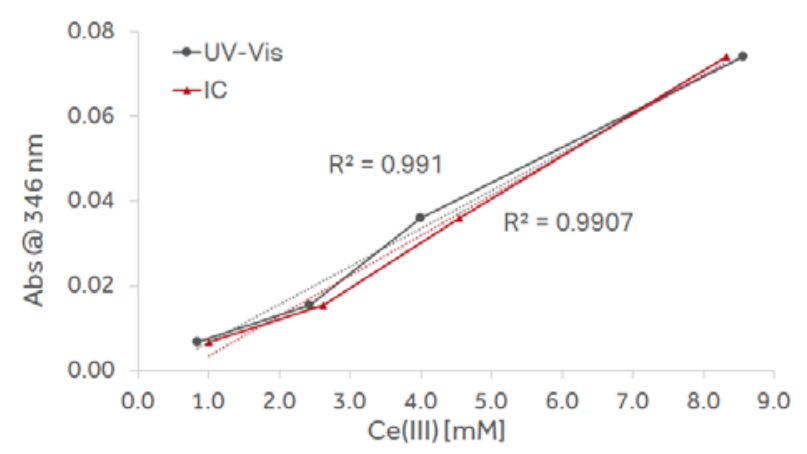

Figure 2. Calibration plots for spectrophotometric measurement of $[\mathrm{Ce}(\mathrm{III})]_{\text {org. }}$.

\section{Discussion}

Previous RDC studies in our laboratory of the kinetics of extraction of $\mathrm{Ce}(\mathrm{III})$ by the TODGA / dodecane system have demonstrated the system to be under MTWCR (mass transport with chemical reaction) control, with rate of extraction being controlled by (i) partitioning of the TODGA into the aqueous phase and (ii) subsequent Ce-TODGA complexation close to the aqueous / organic interface.

More recent RDC experiments with non-equilibrated TODGA, i.e. TODGA that has not been previously contacted / equilibrated with $\mathrm{HNO}_{3}$, demonstrate the simultaneous extraction of both $\mathrm{Ce}$ (III) and $\mathrm{HNO}_{3}$. Relative changes in species concentration in the organic solution phase are detected via UV-Vis spectrophotometry with $\mathrm{HNO}_{3}$ represented by an increase in UV-Vis absorbance at a $\lambda_{\max }$ of $300 \mathrm{~nm}$ and $\mathrm{Ce}(\mathrm{III})$ is represented by an increase in UV-Vis absorbance at a $\lambda_{\max }$ of $346 \mathrm{~nm}$, Figure 3.

While extraction of both species is seen to occur from the onset, a clear difference between the two processes is apparent. Organic $\mathrm{HNO}_{3}$ concentration increases with a linear trend $v s$ time for the duration of the RDC experiments, indicating a constant rate of $\mathrm{HNO}_{3}$ extraction by TODGA with no evidence of saturation within the experimental period. In contrast, organic $\mathrm{Ce}(\mathrm{III})$ concentration increases at an initially steady rate at $\mathrm{t}=<60 \mathrm{~min}$, but begins to plateau as the rate of $\mathrm{Ce}$ extraction slows significantly at $t=>60 \mathrm{~min}$. 


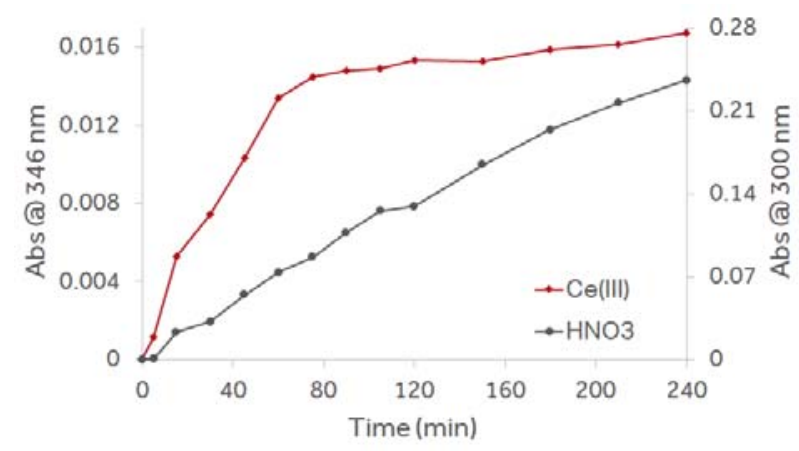

Figure 3. Simultaneous absorbance measurements of $\mathrm{Ce}(\mathrm{III})$ (a) $\mathrm{Abs}^{346} \& \mathrm{HNO}_{3} @ \mathrm{Abs}^{300}$ in the organic phase.

This marked change in the rate of $\mathrm{Ce}$ (III) extraction is not attributable to complete extraction or to having reached the loading capacity of the extractant molecule as significant depletion of the concentration of $\mathrm{Ce}$ (III) in the aqueous phase is not observed and the quantity of TODGA in the RDC system should capably extract all available Ce(III) [7]. Rather, we postulate that the efficiency of the extraction process is inhibited by the increasing organic phase acidity due to extraction of $\mathrm{HNO}_{3}$ from the aqueous solution phase resulting in micellation of the organic extractant molecule.

A number of recent papers discuss the aggregation of TODGA into tetrameric reverse micelles upon contact of the organic phase with $\mathrm{HNO}_{3}$ reporting a critical micellation point at $\mathrm{HNO}_{3}$ concentrations of $\geq 0.7$ $\mathrm{mol} / \mathrm{dm}^{3}$ [8-10]. Our studies demonstrate that a similar organic phase acidity is achieved in the RDC system at $\mathrm{t}$ $=\sim 60 \mathrm{~min}$ through the linear extraction of the 1.0 $\mathrm{mol} / \mathrm{dm}^{3} \mathrm{HNO}_{3}$ aqueous solution phase by TODGA. The resulting aggregation of the extractant molecule and the associated increase in solvent phase viscosity reported in our previous communication [11], negatively impact the mass transfer of the system - causing the plateau in the rate of Ce(III) extraction shown in Figure 3.

To further investigate the effect of solvent acidity on the rates of $\mathrm{Ce}(\mathrm{III})$ and $\mathrm{HNO}_{3}$ extraction, RDC experiments were conducted using TODGA which was pre-equilibrated with $1.0 \mathrm{~mol} / \mathrm{dm}^{3} \mathrm{HNO}_{3}$, and TODGA which was pre-equilibrated with $\mathrm{HNO}_{3}$ and then repeatedly washed with $0.1 \mathrm{~mol} / \mathrm{dm}^{3} \mathrm{NaOH}$ in order to neutralize the acidity of the organic phase.

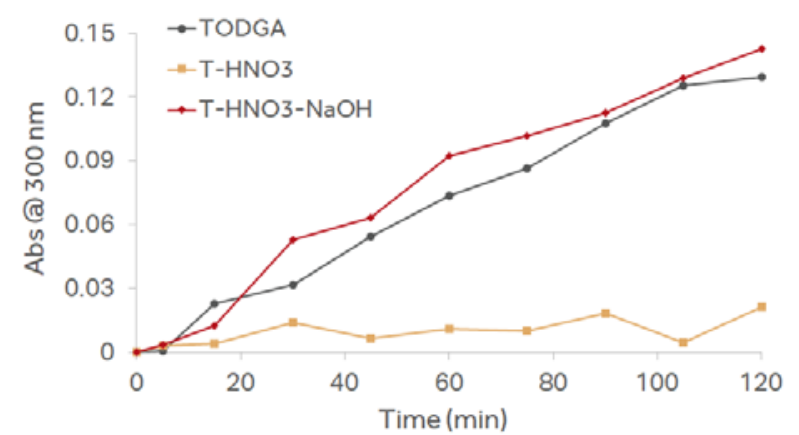

Figure 4. $\mathrm{Abs}^{300}$ change with $\mathrm{HNO}_{3}$ extraction by non-contacted; $\mathrm{HNO}_{3}$-equilibrated \& $\mathrm{HNO}_{3}$ - $\mathrm{NaOH}$-equilibrated TODGA.
In the case of $\mathrm{HNO}_{3}$ extraction, it was observed that very little acid is extracted by the $1 \mathrm{~mol} / \mathrm{dm}^{3}$ $\mathrm{HNO}_{3}$-equilibrated TODGA, as may be expected considering the solvent phase is already at equilibrium with the acidic aqueous phase. In contrast, the $\mathrm{NaOH}$-washed TODGA extracts $\mathrm{HNO}_{3}$ at a rate comparable to that of non-equilibrated TODGA, indicating that the solvent phase acidity established through acidic pre-equilibration has been neutralized, Figure 4.

In the case of $\mathrm{Ce}(\mathrm{III})$ extraction, the influence of acidifying the solvent phase is again demonstrated as the $1 \mathrm{~mol} / \mathrm{dm}^{3} \quad \mathrm{HNO}_{3}$-equilibrated TODGA shows a significant reduction in the initial rate of $\mathrm{Ce}$ (III) extraction when compared to fresh, non-equilibrated TODGA, Figure 5. At $\mathrm{t}=60 \mathrm{~min}, 1.49 \mathrm{mmol} / \mathrm{dm}^{3}$ of $\mathrm{Ce}(\mathrm{III})$ is detected in the non-equilibrated solvent phase while in the $\mathrm{HNO}_{3}$-equilibrated solvent phase, this is reduced by $77.85 \%$ to $0.33 \mathrm{mmol} / \mathrm{dm}^{3}$ of extracted $\mathrm{Ce}(\mathrm{III})$. At $\mathrm{t}=>60 \mathrm{~min}$, the rate of extraction by the non-equilibrated TODGA becomes comparable to that of the $\mathrm{HNO}_{3}$-equilibrated TODGA which continues relatively linearly, extracting a further $0.32 \mathrm{mmol} / \mathrm{dm}^{3}$ of $\mathrm{Ce}(\mathrm{III})$ by $\mathrm{t}=120 \mathrm{~min}$. This supports the theory that the observed plateau in $\mathrm{Ce}$ (III) extraction can be attributed to a critical acidity level in the organic solvent phase.

Neutralization of the acidified solvent by $\mathrm{NaOH}$-wash improves the initial extraction of $\mathrm{Ce}(\mathrm{III})$, extracting 1.88 $\mathrm{mol} / \mathrm{dm}^{3}$ of $\mathrm{Ce}(\mathrm{III})$ by $\mathrm{t}=60 \mathrm{~min}$ at a rate greater than that of the non-equilibrated TODGA. This clearly demonstrates a reversal of the effects of $\mathrm{HNO}_{3}$ pre-equilibration and may also indicate the removal of a small amount of residual acidity in the TODGA remaining from synthesis. However, as seen in Figures 4 $\& 5$, the simultaneous extraction of $\mathrm{HNO}_{3}$ cannot be avoided and the in-process reduction in the rate of $\mathrm{Ce}(\mathrm{III})$ extraction still occurs as solvent phase acidity returns.

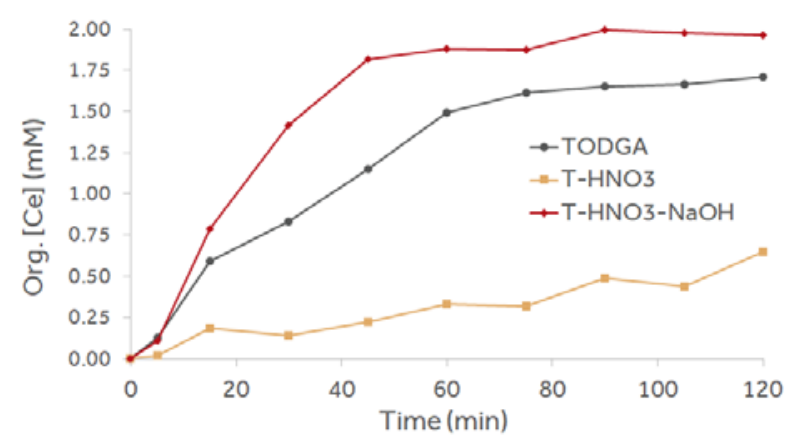

Figure 5. Rate of $\mathrm{Ce}$ (III) extraction by non-equilibrated; $\mathrm{HNO}_{3}$-equilibrated \& $\mathrm{HNO}_{3}-\mathrm{NaOH}$-equilibrated TODGA.

It is clear that the solvent phase acidity, either through pre-contacting with $\mathrm{HNO}_{3}$ or through simultaneous $\mathrm{HNO}_{3}$ extraction, influences the kinetics of $\mathrm{Ce}$ (III) extraction by TODGA in the RDC system. We conclude that, in the context of the MTWCR mechanism described above, $\mathrm{HNO}_{3}$ induced aggregation of the TODGA results in inhibition of both the diffusive mass 
transport of TODGA through the RDC interface membrane into the aqueous solution phase, and the key complexation reaction believed to take place there. While effects of $\mathrm{HNO}_{3}$ pre-equilibration are reversible, TODGA remains susceptible to the in-process acidic environments associated with solvent extraction.

\section{Conclusion}

Comparison of RDC experiments conducted using non-equilibrated \& $\mathrm{HNO}_{3}$-equilibrated TODGA reveals that the rate of $\mathrm{Ce}$ (III) extraction is significantly reduced by the increased solvent phase acidity in the pre-equilibrated experiments as both ligand mass transport to the aqueous phase and subsequent metal-ligand complexation within the system are inhibited by aggregation of the extractant molecule.

While this effect is shown to be reversible through neutralization of the solvent phase prior to use in the RDC, the simultaneous extraction of $\mathrm{HNO}_{3}$ by TODGA means that solvent phase acidity increases in-process until a critical acidity level is reached and extractant aggregation occurs in non-equilibrated and in $\mathrm{NaOH}-$ neutralized TODGA.

In both the pre-equilibrated case and in non-equilibrated and neutralized cases at long extraction times, the net result is a reduction in the $\mathrm{Ce}$ (III) extraction kinetics and continued extraction at a sub-optimal rate. As there is no reason to expect that the thermodynamics of the Ce(III) / TODGA system would be changed then, given adequate time, the distribution values in each case would remain constant though further work may be required in this regard.

This study offers important information regarding the optimal use of TODGA in nuclear waste management processes where the extractant is expected to perform under acidic conditions. Clearly, in an acidic solvent phase, the extraction kinetics of TODGA are non-optimal and may reduce overall process efficiency.

Furthermore, the viscosity increase and change in surface tension associated with solvent phase acidity and extractant aggregation may influence the efficiency of other plants, such as centrifugal contactors, where a change in droplet size and viscous drag could again result in a reduction in efficiency of the system. In any reprocessing system, it is desirable to operate in the most effective and efficient conditions possible in order that the time, energy consumption and cost may be kept to a minimum and hence further investigation into the effects of solvent acidity on solvent extraction kinetics are warranted.

\section{Acknowledgements}

$\mathrm{MB}$ is supported by (i) the European Commission under the Euratom Nuclear Fission and Radiation Protection Theme of the $7^{\text {th }}$ Framework Programme for
Research \& Technological Development (FP7 EURATOM Fission-2012-2.3.1 Project SACSESS, Ref. 323282) \& (ii) the EPSRC (award no. EP/L018616/1, "PACIFIC - Providing a Nuclear Fuel Cycle in the UK for Implementing Carbon Reductions). CB is supported by the Lloyd's Register Foundation (LRF). The LRF supports the advancement of engineering-related education, and funds research and development that enhances safety of life at sea, on land and in the air.

\section{References}

[1] G. Modolo, A. Wilden, P. Kaufholz, D. Bosbach, and A. Geist, Development and demonstration of innovative partitioning processes (i-sanex and 1-cycle sanex) for actinide partitioning, Progress in Nuclear Energy, 72 (2014), pp. 107-114.

[2] W.J. Albery, J.F. Burke, E.B. Leffler and J. Hadgraft, Interfacial transfer studied with a rotating diffusion cell, J. Chem. Soc., Faraday Trans 1, 72 (1976), pp. 1618-1626.

[3] W.J. Albery and R.A. Choudhery, Transport and kinetics in reactions involving two liquid phases, $J$. Phys. Chem., 92 (1988), pp. 1151-1155.

[4] J.P. Simonin, C. Boxall and A.M. Lelias, SACSESS deliverable d12.5 - kinetics of extr. And stripping, A. Geist and S. Bourg, Eds. (2015).

[5] M.A. Bromley and C. Boxall, A study of cerium extraction by tbp and todga using a rotating diffusion cell, Nukleonika, 60 (Pt II), (2015), pp. 859-864.

[6] C. Boxall, Add. To SACSESS deliverable d12.5 kinetics of extr. And stripping, A. Geist and S. Bourg, Eds. (2015).

[7] E. Metwally, A.S. Saleh, S.M. Abdel-Wahaab and H.A. El-Naggar, Extraction behavior of cerium by tetraoctyldiglycolamide from nitric acid solutions, J Radioanal Nucl Chem, 286 (2010), pp. 217-221.

[8] S. Nave, G. Modolo, C. Madic and F. Testard, Aggregation properties of n,n, n', $n^{\prime}$-tetraoctyl-3oxapentanediamide (todga) in n-dodecane, Solvent Extr. Ion Exch., 22, (2004), pp. 527-551.

[9] M.P. Jensen, T. Yaita and R. Chiarizia, Reverse-micelle formation in the partitioning of trivalent f-element cations by biphasic systems containing a tetraalkyldiglycolamide, Langmuir, 23 (2007), pp. 4765-4774.

[10]R. Ganguly, J.N. Sharma and N. Choudhury, Todga based w/o microemulsion in dodecane: An insight into the micellar aggregation characteristics by dynamic light scattering and viscometry, $J$. Colloid Interface Sci., 355 (2011), pp. 458-463.

[11]M.A. Bromley and C. Boxall, The effects of nitric acid on extraction properties of todga during fission product management, MRS Advances, 2 (2016), pp. 563-568. 